\title{
THREE-DIMENSIONAL OSCILLATING FLOW BETWEEN TWO PARALLEL PLATES THROUGH A POROUS MEDIUM
}

\author{
M. GURIA* \\ Department of Mathematics \\ Ghatal Rabindra Satabarsiki Mahavidyalaya \\ Paschim Medinipur, West Bengal, INDIA \\ E-mail: mrinmoy9832@yahoo.com \\ N. GHARA and R.N. JANA \\ Department of Applied Mathematics \\ with Oceanology and Computer programming \\ Vidyasagar University \\ Midnapore 721102 INDIA
}

\begin{abstract}
An unsteady Couette flow between two parallel plates when upper plates oscillates in its own plane and is subjected to a constant suction and the lower plate to a injection velocity distribution through the porous medium has been analyzed. The approximate solution has been obtained using perturbation technique. It is seen that the primary velocity increases whereas the secondary velocity decreases with an increase in permeability parameter. It is also found that the primary velocity increases with an increase in the Reynolds number as well as the suction parameter. The magnitude of the secondary velocity increases near the stationary plate but decreases near the oscillating plate with an increase in the Reynolds number. Whereas, it increases with an increase in the suction parameter.
\end{abstract}

Key words: Couette flow, three-dimensional, injection, suction, transverse sinusoidal.

\section{Introduction}

The unsteady boundary layer flow has been the subject of intensive studies in the last two decades, deals with the boundary layer responses to imposed oscillations. The oscillatory flow and heat transfer problems are important in view of their applications to astrophysics, geophysics and engineering. Fluid through a porous medium is important in many branches of science and technology. The study of such flows was first initiated by Lighthill (1954) who studied the effect of free stream oscillations on the boundary layer flow of a viscous, incompressible fluid past an infinite plate. Varshney (1979) discussed an oscillatory twodimensional flow through porous medium bounded by a horizontal porous plate subjected to a variable suction velocity. Rapits (1983) analysed the unsteady flow through a porous medium bounded by an infinite porous plate subjected to a constant suction and variable temperature.Rapits and Perdikis (1985) further studied the problem of a free convective flow through a porous medium bounded by a vertical porous plate with constant suction when the free stream velocity oscillates in time about a constant mean value. Singh and Sharma (2001) studied the three-dimensional Couette flow through a porous medium. Singh and Sharma (2002) also studied the effect of periodic permeability on three-dimensional convective flow and heat transfer through a porous medium.

The objective of this paper is to study the effect of permeability on a three dimensional Couette flow when the upper plates oscillates in its own plane and is subjected to a constant suction and the lower plate to

\footnotetext{
* To whom correspondence should be addressed
} 
a injection velocity distribution. It is seen that the primary velocity increases whereas the secondary velocity decreases with an increase in the permeability parameter. It is found that the primary velocity increases with an increase in the Reynolds number as well as the suction parameter. The magnitude of the secondary velocity increases near the stationary plate but decreases near the oscillating plate with an increase in the Reynolds number, whereas it increases with an increase in the suction parameter.

\section{Basic equations and solution}

Consider the unsteady flow of a viscous incompressible fluid between two infinite parallel flat porous plates through a porous medium. Let $d$ be the distance between two plates. The upper plate oscillates in its own plane with velocity distribution of the form

$$
u^{*}=U\left[1+\varepsilon e^{i \omega^{*} t^{*}}\right]
$$

where $\varepsilon(\prec \prec 1)$ is the amplitude of the oscillation, $\omega^{*}$ is the frequency of the oscillations, $t^{*}$ is the time and $U$ is the free stream velocity. We choose the $x^{*}-$ axis in the direction of the flow, $y^{*}-$ axis perpendicular to the direction of the flow and $z^{*}$ - axis normal to the $x^{*} y^{*}$ - plane, lying in the plane of the lower plate which is assumed to be at rest. The upper plate is subjected to a constant suction $V_{0}$ and the lower plate to a transverse sinusoidal injection velocity distribution of the form

$$
v^{*}=-V_{0}\left[1+\varepsilon \cos \left(\frac{\pi z^{*}}{d}\right)\right] .
$$

Let $u^{*}, v^{*}, w^{*}$ be the velocity components in the directions of $x^{*}-, y^{*}-$, and $z^{*}-$ axes respectively, then the problem is governed by the following equations

$$
\begin{aligned}
& \frac{\partial v^{*}}{\partial y^{*}}+\frac{\partial w^{*}}{\partial z^{*}}=0, \\
& \frac{\partial u^{*}}{\partial t^{*}}+v^{*} \frac{\partial u^{*}}{\partial y^{*}}+w^{*} \frac{\partial u^{*}}{\partial z^{*}}=v\left(\frac{\partial^{2} u^{*}}{\partial y^{* 2}}+\frac{\partial^{2} u^{*}}{\partial z^{* 2}}\right)-\frac{v u^{*}}{K^{*}}, \\
& \frac{\partial v^{*}}{\partial t^{*}}+v^{*} \frac{\partial v^{*}}{\partial y^{*}}+w^{*} \frac{\partial v^{*}}{\partial z^{*}}=-\frac{1}{\rho} \frac{\partial p}{\partial y}+v\left(\frac{\partial^{2} v^{*}}{\partial y^{* 2}}+\frac{\partial^{2} v^{*}}{\partial z^{* 2}}\right)-\frac{v v^{*}}{K^{*}}, \\
& \frac{\partial w^{*}}{\partial t^{*}}+v^{*} \frac{\partial w^{*}}{\partial y^{*}}+w^{*} \frac{\partial w^{*}}{\partial z^{*}}=-\frac{1}{\rho} \frac{\partial p}{\partial z}+v\left(\frac{\partial^{2} w^{*}}{\partial y^{* 2}}+\frac{\partial^{2} w^{*}}{\partial z^{* 2}}\right)-\frac{v w^{*}}{K^{*}}
\end{aligned}
$$

where $v$ is the kinematic coefficient of viscosity, $\rho$ is the density, $p^{*}$ is the fluid pressure, $K^{*}$ is the permeability of the porous medium.

The boundary conditions of the problem are 


$$
\begin{aligned}
& u^{*}=0, \quad v^{*}=-V_{0}\left[1+\varepsilon \cos \left(\frac{\pi z^{*}}{d}\right)\right], \quad w^{*}=0 \quad \text { at } \quad y^{*}=0, \\
& u^{*}=U\left[1+\varepsilon e^{i \omega^{*} t^{*}}\right], \quad v^{*}=-V_{0}, \quad w^{*}=0 \quad \text { at } \quad y^{*}=d .
\end{aligned}
$$

Introducing the non-dimensional variables

$$
y=\frac{y^{*}}{d}, \quad z=\frac{z^{*}}{d}, \quad t=c t^{*}, \quad p=\frac{p^{*}}{\rho U^{2}}, \quad u=\frac{u^{*}}{U}, \quad v=\frac{v^{*}}{U}, \quad w=\frac{w^{*}}{U} .
$$

Equations (2.3)-(2.6) become

$$
\begin{aligned}
& \frac{\partial v}{\partial y}+\frac{\partial w}{\partial z}=0 \\
& \omega \frac{\partial u}{\partial t}+\operatorname{Re}\left(v \frac{\partial u}{\partial y}+w \frac{\partial u}{\partial z}\right)=\frac{\partial^{2} u}{\partial y^{2}}+\frac{\partial^{2} u}{\partial z^{2}}-\frac{u}{K}, \\
& \omega \frac{\partial v}{\partial t}+\operatorname{Re}\left(v \frac{\partial v}{\partial y}+w \frac{\partial v}{\partial z}\right)=-\operatorname{Re} \frac{\partial p}{\partial y}+\left(\frac{\partial^{2} v}{\partial y^{2}}+\frac{\partial^{2} v}{\partial z^{2}}\right)-\frac{v}{K}, \\
& \omega \frac{\partial w}{\partial t}+\operatorname{Re}\left(v \frac{\partial w}{\partial y}+w \frac{\partial w}{\partial z}\right)=-\operatorname{Re} \frac{\partial p}{\partial z}+\left(\frac{\partial^{2} w}{\partial y^{2}}+\frac{\partial^{2} w}{\partial z^{2}}\right)-\frac{w}{K}
\end{aligned}
$$

where $\operatorname{Re}=U d / v$, the Reynolds number, $S=V_{0} / U$, the suction parameter and $\omega=\omega^{*} d^{2} / \nu$, the frequency parameter, $K=K^{*} / d^{2}$, the permeability parameter. Using Eqs (2.8), the boundary conditions Eqs (2.7) become

$$
\begin{aligned}
& u=0, \quad v=-S[1+\varepsilon \cos (\pi z)], \quad w=0 \quad \text { at } \quad y=0, \\
& u=\left(1+\varepsilon e^{i \omega t}\right), \quad v=-S, \quad w=0 \quad \text { at } \quad y=1 .
\end{aligned}
$$

\section{Solution}

In order to solve the differential Eqs (2.9)-(2.12), we assume the solution of the following form

$$
\begin{aligned}
& u(y, z, t)=u_{0}(y)+\varepsilon u_{1}(y, z, t)+\varepsilon^{2} u_{2}(y, z, t)+\cdots, \\
& v(y, z, t)=v_{0}(y)+\varepsilon v_{1}(y, z, t)+\varepsilon^{2} v_{2}(y, z, t)+\cdots, \\
& w(y, z, t)=w_{0}(y)+\varepsilon w_{1}(y, z, t)+\varepsilon^{2} w_{2}(y, z, t)+\cdots, \\
& p(y, z, t)=p_{0}(y)+\varepsilon p_{1}(y, z, t)+\varepsilon^{2} p_{2}(y, z, t)+\cdots .
\end{aligned}
$$


Assume $w_{0}=0$ and $p_{0}=$ constant. On substituting Eq.(3.1) in Eqs (2.9)-(2.12) and equating the term free from $\varepsilon$, we get the following system of differential equations

$$
\begin{aligned}
& v_{0}^{\prime}=0, \\
& u_{0}^{\prime \prime}+S \operatorname{Re} u_{0}^{\prime}-\frac{u_{0}}{K}=0
\end{aligned}
$$

where primes denote differentiation with respect to $y$ and the corresponding boundary conditions become

$$
u_{0}=0, \quad v_{0}=-S \quad \text { at } \quad y=0, \quad \text { and } \quad u_{0}=1, \quad v_{0}=-S \quad \text { at } \quad y=1 .
$$

The solutions of Eqs (3.2) and (3.3), using Eq.(3.4) are

$$
v_{0}[y]=-S, \quad\left(u_{0} y\right)=\frac{\left(e^{-n_{1} y}-e^{-n_{2} y}\right)}{\left(e^{-n_{1}}-e^{-n_{2}}\right)}
$$

where

$$
n_{1,2}=\frac{1}{2}\left[S \operatorname{Re} \pm\left\{S^{2} \operatorname{Re}^{2}+4 / K\right\}^{1 / 2}\right]
$$

On substituting Eqs (3.1) in Eqs (2.9)-(2.12) and equating the coefficient of $\varepsilon$, we get the following system of differential equations

$$
\begin{aligned}
& \frac{\partial v_{1}}{\partial y}+\frac{\partial w_{1}}{\partial z}=0 \\
& \frac{\partial u_{1}}{\partial t}+\operatorname{Re}\left(-S \frac{\partial u_{1}}{\partial y}+v_{1} \frac{\partial u_{0}}{\partial y}\right)=\frac{\partial^{2} u_{1}}{\partial y^{2}}+\frac{\partial^{2} u_{1}}{\partial z^{2}}-u_{1} / K \\
& \omega \frac{\partial v_{1}}{\partial t}-S \operatorname{Re} \frac{\partial v_{1}}{\partial y}=-\operatorname{Re} \frac{\partial p_{1}}{\partial y}+\frac{\partial^{2} v_{1}}{\partial y^{2}}+\frac{\partial^{2} v_{1}}{\partial z^{2}}-v_{1} / K \\
& \omega \frac{\partial w_{1}}{\partial t}-S \operatorname{Re} \frac{\partial w_{1}}{\partial y}=-\operatorname{Re} \frac{\partial p_{1}}{\partial z}+\frac{\partial^{2} w_{1}}{\partial y^{2}}+\frac{\partial^{2} w_{1}}{\partial z^{2}}-w_{1} / K
\end{aligned}
$$

The corresponding boundary conditions become

$$
\begin{aligned}
& u_{1}=0, \quad v_{1}=-S \cos (\pi z), \quad w_{1}=0 \quad \text { at } \quad y=0, \\
& u_{1}=e^{i \omega t}, \quad v_{1}=0, \quad w_{1}=0 \quad \text { at } \quad y=1 .
\end{aligned}
$$


These are the linear partial differential equations describing the three-dimensional flow. To solve Eqs (3.8)-(3.10), we assume velocity components and pressure as

$$
\begin{aligned}
& u_{1}(y, z, t)=u_{11}(y) \cos (\pi z)+u_{12}(y) e^{i \omega t}, \\
& v_{1}(y, z, t)=v_{11}(y) \cos (\pi z)+v_{12}(y) e^{i \omega t}, \\
& w_{1}(y, z, t)=-\left[\frac{1}{\pi} v_{11}^{\prime}(y) \sin (\pi z)+z v_{12}^{\prime}(y) e^{i \omega t}\right], \\
& p_{1}(y, z, t)=p_{11}(y) \cos (\pi z)+p_{12}(y) e^{i \omega t} .
\end{aligned}
$$

Substituting Eqs (3.12)-(3.15) in Eqs (3.8)-(3.10) and comparing the coefficients of harmonic terms, we obtain the following set of differential equations

$$
\begin{aligned}
& u_{11}^{\prime \prime}+S \operatorname{Re} u_{11}^{\prime}-\left(\pi^{2}+\frac{1}{K}\right) u_{11}=\operatorname{Re} u_{0}^{\prime} v_{11}, \\
& u_{12}^{\prime \prime}+S \operatorname{Re} u_{12}^{\prime}-\left(i \omega+\frac{1}{K}\right) u_{12}=\operatorname{Re} u_{0}^{\prime} v_{12}, \\
& v_{11}^{\prime \prime}+S \operatorname{Re} v_{11}^{\prime}-\left(\pi^{2}+\frac{1}{K}\right) v_{11}=\operatorname{Re} p_{11}^{\prime}, \\
& v_{12}^{\prime \prime}+S \operatorname{Re} v_{12}^{\prime}-\left(i \omega+\frac{1}{K}\right) v_{12}=\operatorname{Re} p_{12}^{\prime}, \\
& v_{11}^{\prime \prime \prime}+S \operatorname{Re} v_{11}^{\prime \prime}-\left(\pi^{2}+\frac{1}{K}\right) v_{11}^{\prime}=\operatorname{Re} \pi^{2} p_{11}, \\
& v_{12}^{\prime \prime \prime}+S \operatorname{Re} v_{12}^{\prime \prime}-\left(i \omega+\frac{1}{K}\right) v_{12}^{\prime}=0 .
\end{aligned}
$$

When $K \rightarrow \infty$, Eqs (3.16)-(3.21) coincide with Eqs (2.13)-(3.2) of Guria et al. (2007). The corresponding boundary conditions are

$$
\begin{aligned}
& u_{11}=0, \quad u_{12}=0, \quad v_{11}=-S, \quad v_{12}=0, \quad v_{11}^{\prime}=0, \quad v_{12}^{\prime}=0 \quad \text { at } \quad y=0 \text {, } \\
& u_{11}=0, \quad u_{12}=1, \quad v_{11}=0, \quad v_{12}=0, \quad v_{11}^{\prime}=0, \quad v_{12}^{\prime}=0 \quad \text { at } \quad y=1 \text {. }
\end{aligned}
$$

Solving Eqs (3.16)-(3.21) under boundary conditions Eqs (3.22) and on using Eqs (3.12)-(3.15), we get 


$$
\begin{aligned}
& u_{1}(y, z, t)=\left[C_{9} e^{-r_{1} y}+C_{10} e^{-r_{2} y}+C_{1} e^{-\left(r_{1}+n_{1}\right) y}+C_{2} e^{-\left(r_{2}+n_{1}\right) y}+C_{3} e^{\left(\pi-n_{1}\right) y}+\right. \\
& +C_{4} e^{-\left(\pi+n_{1}\right) y}+C_{5} e^{-\left(r_{1}+n_{2}\right) y}+C_{6} e^{-\left(r_{2}+n_{2}\right) y}+C_{7} e^{\left(\pi-n_{2}\right) y}+ \\
& \left.+C_{8} e^{-\left(\pi+n_{2}\right) y}\right] \cos \pi z+\frac{\left(e^{-m_{1} y}-e^{-m_{2} y}\right)}{\left(e^{-m_{1}}-e^{-m_{2}}\right)} e^{i \omega t}, \\
& v_{1}(y, z)=\left[A e^{-r_{1} y}+B e^{-r_{2} y}+C e^{\pi y}+D e^{-\pi y}\right] \cos (\pi z), \\
& w_{l}(y, z)=\frac{1}{\pi}\left[A r_{1} e^{-r_{1} y}+B r_{2} e^{-r_{2} y}-C \pi e^{\pi y}+D \pi e^{-\pi y}\right] \sin (\pi z), \\
& p_{1}(y, z)=S\left[\left\{C e^{\pi y}+D e^{-\pi y}\right\}-\frac{1}{K \pi R e}\left\{C e^{\pi y}-D e^{-\pi y}\right\}\right] \cos (\pi z)
\end{aligned}
$$

where

$$
\begin{aligned}
& K_{1}=\frac{\operatorname{Re}}{\left(e^{-n_{1}}-e^{-n_{2}}\right)}, \quad C_{1}=\frac{-A K_{1} n_{1}}{\left(2 r_{1} n_{1}+1 / K\right)}, \quad C_{2}=\frac{-B K_{1} n_{1}}{\left(2 r_{2} n_{1}+1 / K\right)}, \\
& C_{3}=\frac{-C K_{1} n_{1}}{\pi\left(S \operatorname{Re}-2 n_{1}\right)}, \quad C_{4}=\frac{D K_{1} n_{1}}{\pi\left(S \operatorname{Re}-2 n_{1}\right)}, \quad C_{5}=\frac{K_{1} A n_{2}}{\left(2 r_{1} n_{2}+1 / K\right)}, \\
& C_{6}=\frac{K_{1} B n_{2}}{\left(2 r_{2} n_{2}+1 / K\right)}, \quad C_{7}=\frac{C K_{1} n_{2}}{\pi\left(S \operatorname{Re}-2 n_{2}\right)}, \quad C_{8}=\frac{D K_{1} n_{2}}{-\pi\left(S \operatorname{Re}-2 n_{2}\right)}, \\
& m_{1,2}=\frac{1}{2}\left[S \operatorname{Re} \pm\left\{S^{2} \operatorname{Re}^{2}+4\left(i \omega+\frac{1}{K}\right)\right\}^{1 / 2}\right], \\
& r_{1,2}=\frac{1}{2}\left[S \operatorname{Re} \pm\left\{S^{2} \operatorname{Re}^{2}+4\left(\pi^{2}+\frac{1}{K}\right)\right\}\right], \\
& C_{9}=\frac{1}{\left(e^{-r_{2}}-e^{\left.-r_{1}\right)}\left[C_{1}\left\{e^{-\left(r_{1}+n_{1}\right) y}-e^{-r_{2}}\right\}+C_{2}\left\{e^{-\left(r_{2}+n_{1}\right) y}-e^{-r_{2}}\right\}+\right.\right.} \\
& +C_{3}\left\{e^{\left(\pi-n_{1}\right) y}-e^{-r_{2}}\right\}+C_{4}\left\{e^{-\left(\pi+n_{1}\right) y}-e^{-r_{2}}\right\}+C_{5}\left\{e^{-\left(r_{1}+n_{2}\right) y}-e^{-r_{2}}\right\}+ \\
& \left.+C_{6}\left\{e^{-\left(r_{2}+n_{2}\right) y}-e^{-r_{2}}\right\}+C_{7}\left\{e^{\left(\pi-n_{2}\right) y}-e^{-r_{2}}\right\}+C_{8}\left\{e^{-\left(\pi+n_{2}\right) y}-e^{-r_{2}}\right\}\right],
\end{aligned}
$$




$$
\begin{aligned}
& C_{10}=\frac{-1}{\left(e^{-r_{2}}-e^{-r_{I}}\right)}\left[C_{1}\left\{e^{-\left(r_{1}+n_{1}\right) y}-e^{-r_{I}}\right\}+C_{2}\left\{e^{-\left(r_{2}+n_{1}\right) y}-e^{-r_{I}}\right\}+\right. \\
& +C_{3}\left\{e^{\left(\pi-n_{1}\right) y}-e^{-r_{I}}\right\}+C_{4}\left\{e^{-\left(\pi+n_{1}\right) y}-e^{-r_{I}}\right\}+C_{5}\left\{e^{-\left(r_{1}+n_{2}\right) y}-e^{-r_{I}}\right\}+ \\
& \left.+C_{6}\left\{e^{-\left(r_{2}+n_{2}\right) y}-e^{-r_{1}}\right\}+C_{7}\left\{e^{\left(\pi-n_{2}\right) y}-e^{-r_{I}}\right\}+C_{8}\left\{e^{-\left(\pi+n_{2}\right) y}-e^{-r_{I}}\right\}\right] .
\end{aligned}
$$

When $K \rightarrow \infty$ the solutions coincide with the solution of Guria et al. (2007). The solution also exists for the blowing at the plate.

\section{Results and discussion}

We have presented the non-dimensional velocities $u$ and $w$ for different values of the permeability parameter $K$, Reynolds number Re and suction parameter $S$ in Figs 1-5. In Fig.1 we have presented the primary velocity for several values of the permeability parameter $K$. It is seen the primary velocity increases withan increase in $K$. The effects of the Reynolds number and suction parameter on the primary velocity are shown in Figs 2 and 3. It is observed that the primary velocity increases with an increase either in the Reynolds number Re or suction parameter $S$.

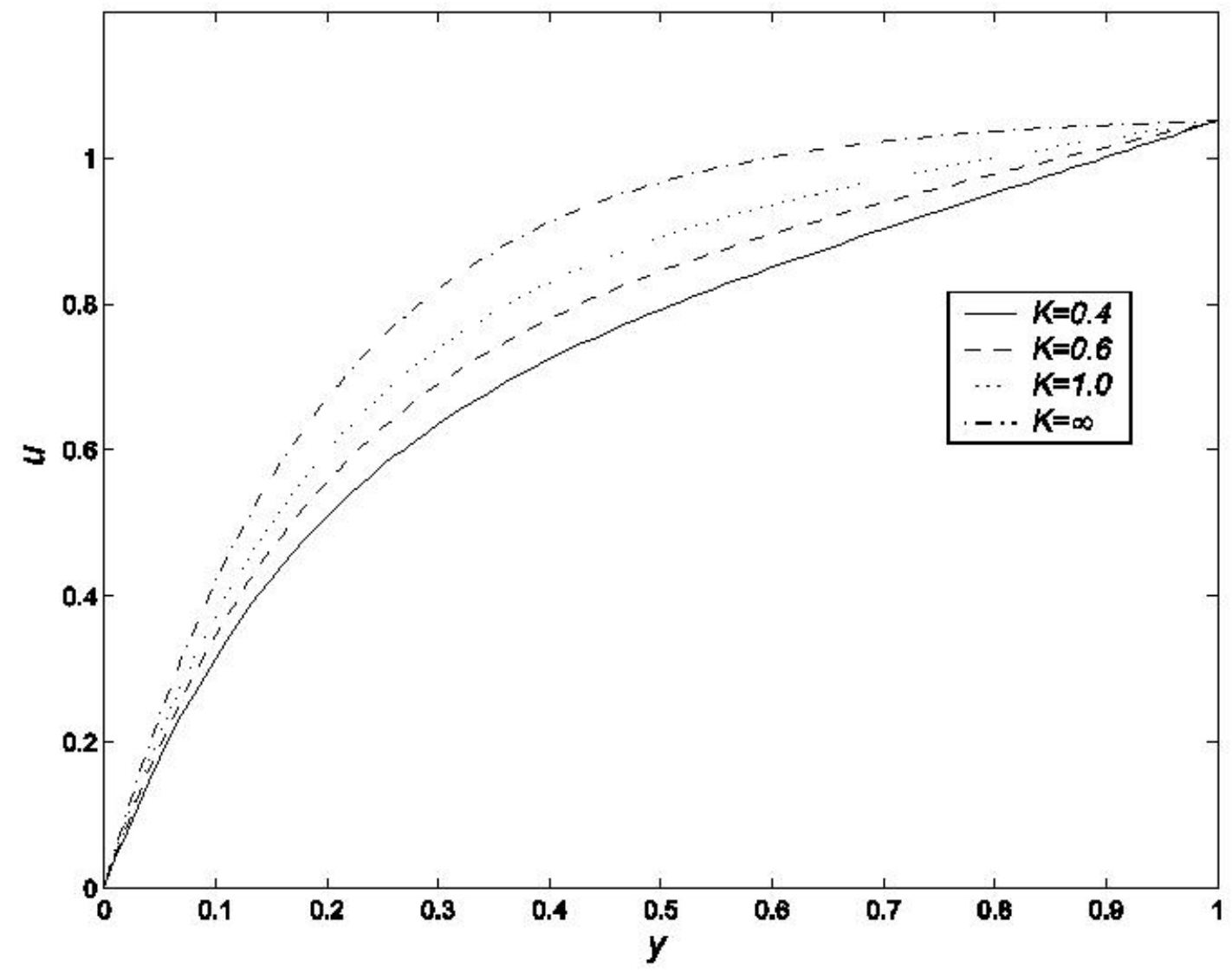

Fig.1. Primary velocity for $\omega t=0, S=1.0, t=0.2, \omega=5, \operatorname{Re}=5.0, \varepsilon=0.05$. 


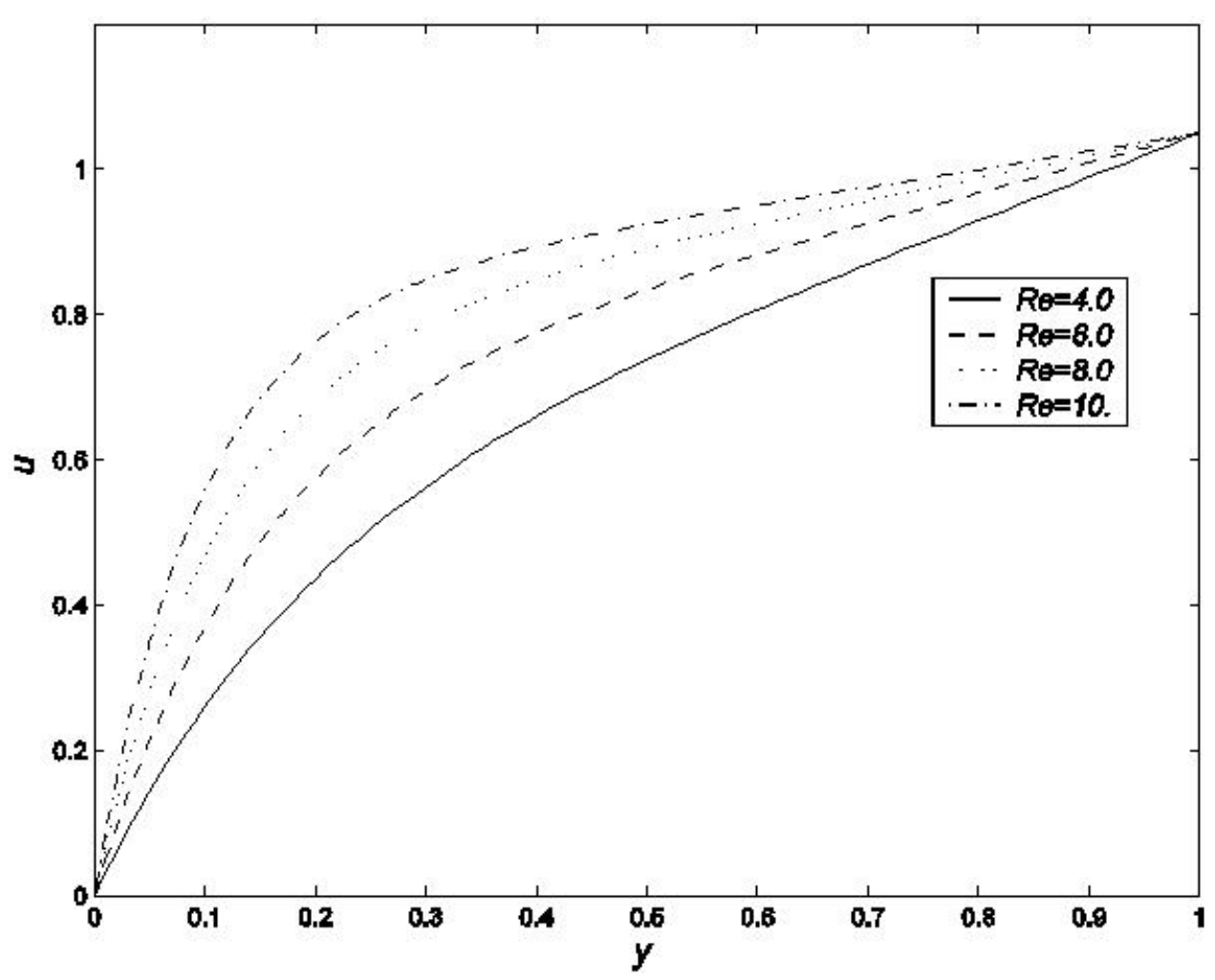

Fig.2. Primary velocity for $\omega t=0, S=1.0, t=0.2, \omega=5, K=0.4, \varepsilon=0.05$.

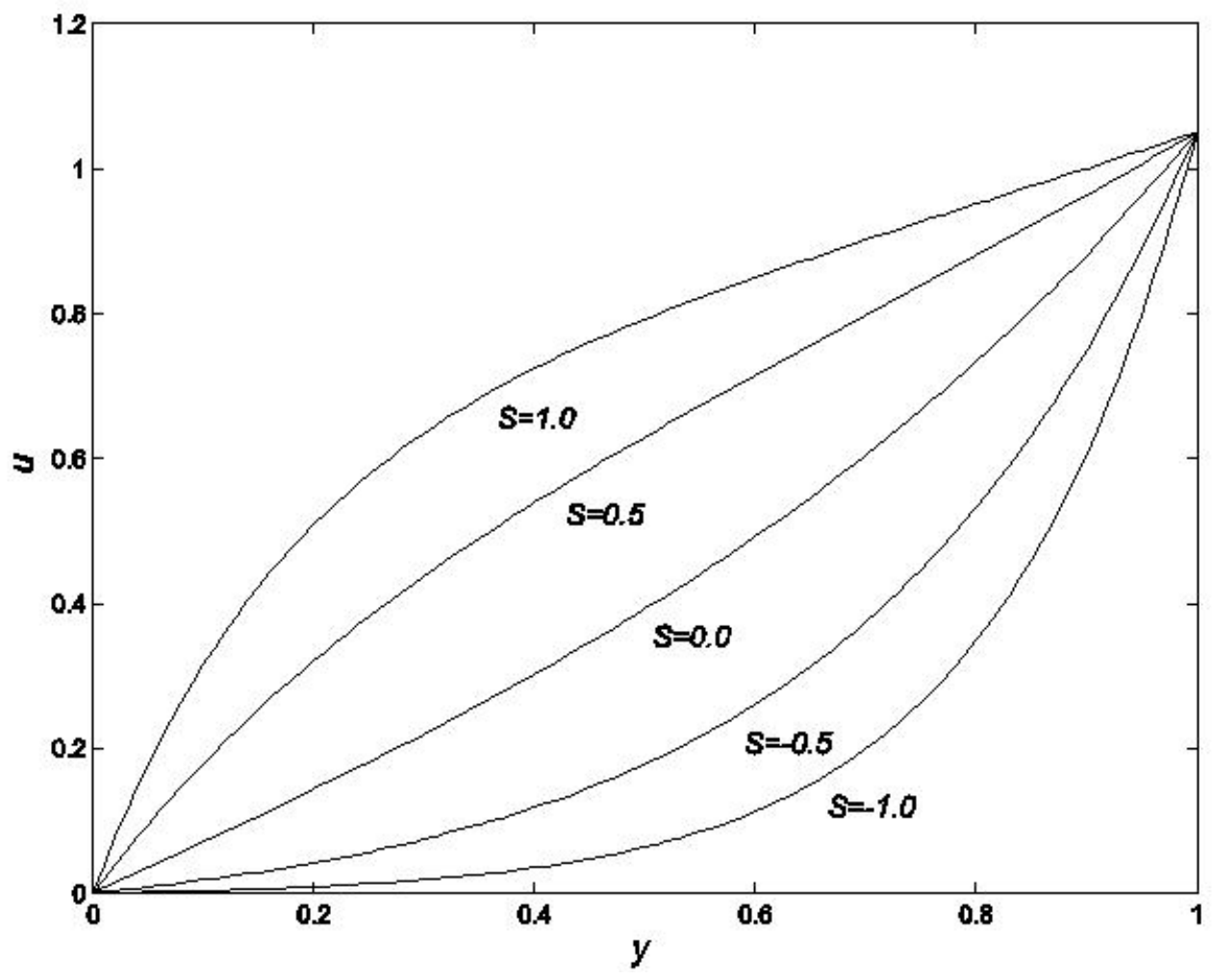

Fig.3. Primary velocity for $\omega t=0, \operatorname{Re}=5.0, K=0.4, z=0.0, t=0.2, \omega=5, \varepsilon=0.05$. 


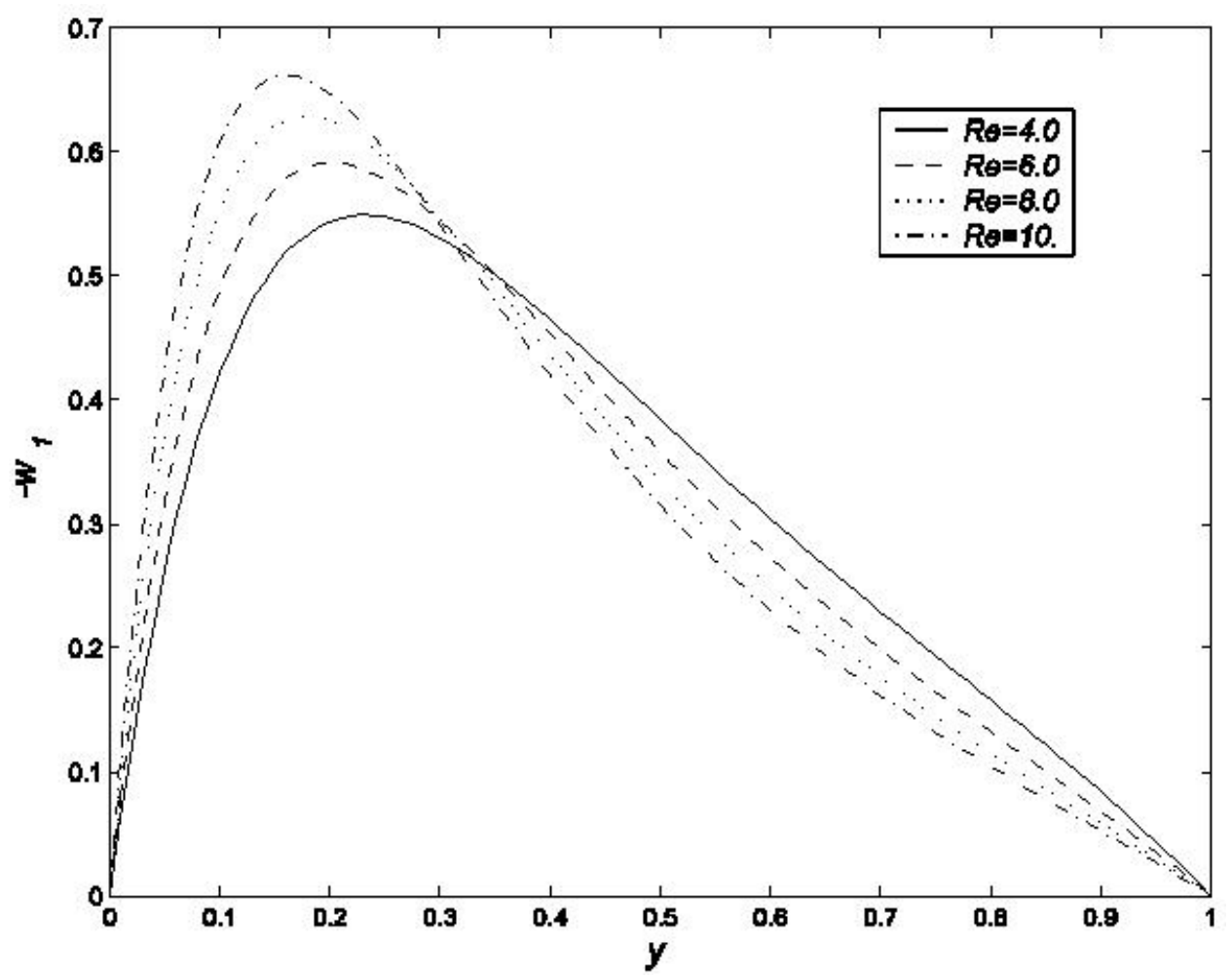

Fig.4. Secondary velocity profile $-w_{1}$ for $K=0.4, \omega=5.0, S=1.0$.

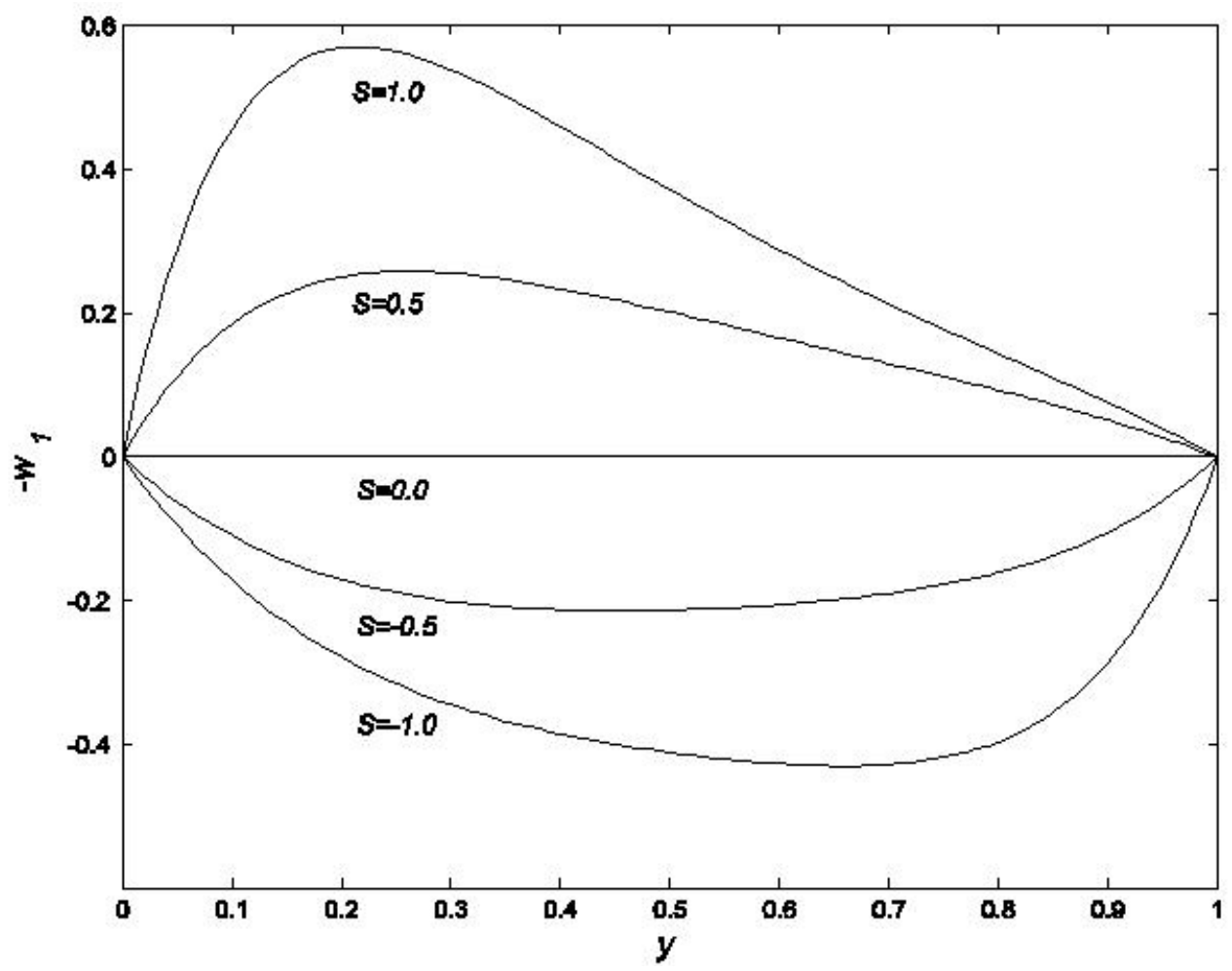

Fig.5. Secondary velocity profile $-w_{1}$ for $K=0.4, \omega=5.0, \mathrm{Re}=5.0$. 
The effect of the permeability parameter on the secondary velocity is shown in Tab.1. It is observed from Tab. 1 that the secondary velocity decreases near the plate and increases away from the plate with an increase in the permeability parameter $K$.

Table 1 . Secondary velocity $-w_{1}$ for $z=0.5, \operatorname{Re}=5$ and $\omega=10$.

\begin{tabular}{|l|l|l|l|l|}
\hline$y \backslash K$ & 0.2 & 0.6 & 1.0 & $\infty$ \\
\hline 0.0 & 0.00000000 & 0.00000024 & 0.00000000 & 0.00000000 \\
\hline 0.2 & 0.57268330 & 0.56783800 & 0.56680730 & 0.56521980 \\
\hline 0.4 & 0.45605160 & 0.46108120 & 0.46213280 & 0.46373890 \\
\hline 0.6 & 0.28446830 & 0.28877630 & 0.28968340 & 0.29107200 \\
\hline 0.8 & 0.14370380 & 0.14388940 & 0.14393010 & 0.14398950 \\
\hline 1.0 & 0.00000417 & -0.00000067 & 0.00000070 & -0.00000367 \\
\hline
\end{tabular}

The variations of the secondary velocity for different values of the suction parameter $S$ and Reynolds number Re are shown in Figs 4 and 5. The magnitude of the secondary velocity increases near the lower plate and decreases away from the lower plate with an increase in the Reynolds number. The magnitude of the secondary velocity increases with an increase in the suction parameter.

Knowing the velocity field it is interesting to know the shear stress at the plates. The nondimensional shear stress at the plate due to the primary flow is given by

$$
\tau_{x}=\left(\frac{\partial u}{\partial y}\right)_{y=0}=u_{0}^{\prime}(0)+\varepsilon u_{l}^{\prime}(0) .
$$

In terms of the amplitude and phase shift, the shear stress due to the primary flow can be expressed as

$$
\tau_{x}=u_{0}^{\prime}(0)+\varepsilon u_{11}^{\prime}(0) \cos \pi z+\varepsilon|R| \cos (\omega t+\varphi)
$$

where

$$
|R|=\sqrt{t_{r}^{\prime 2}+t_{i}^{\prime 2}} \tan \varphi=\frac{t_{i}^{\prime}}{t_{r}^{\prime}}
$$

The amplitude $|R|$ and phase shift $\tan \varphi$ of the shear stress due to the primary flow are shown in Figs 6 and 7 for different values of $K$ against Re. It is seen that the amplitude increases with an increase in either $K$ or Re. The magnitude of the phase shift decreases with an increase in the Reynolds number but increases with an increase in the permeability parameter.

The non-dimensional shear stress due to the secondary flow can be expressed as

$$
\tau_{z}=\left(\frac{\partial w}{\partial y}\right)_{y=0}=w_{0}^{\prime}(0)+\varepsilon w_{l}^{\prime}(0),=\left|R_{2}\right| \sin (\pi z)
$$

where

$$
R_{2}=-A r_{1}^{2} / \pi-B r_{2}^{2} / \pi-C \pi-D \pi
$$

The shear stress due to the secondary flow is plotted in terms of $\left|R_{2}\right|$ against the Reynolds number for different values of the permeability parameter in Fig.8. It is seen that $\left|R_{2}\right|$ decreases with an increase in the permeability parameter but increases with an increase in the Reynolds number. 


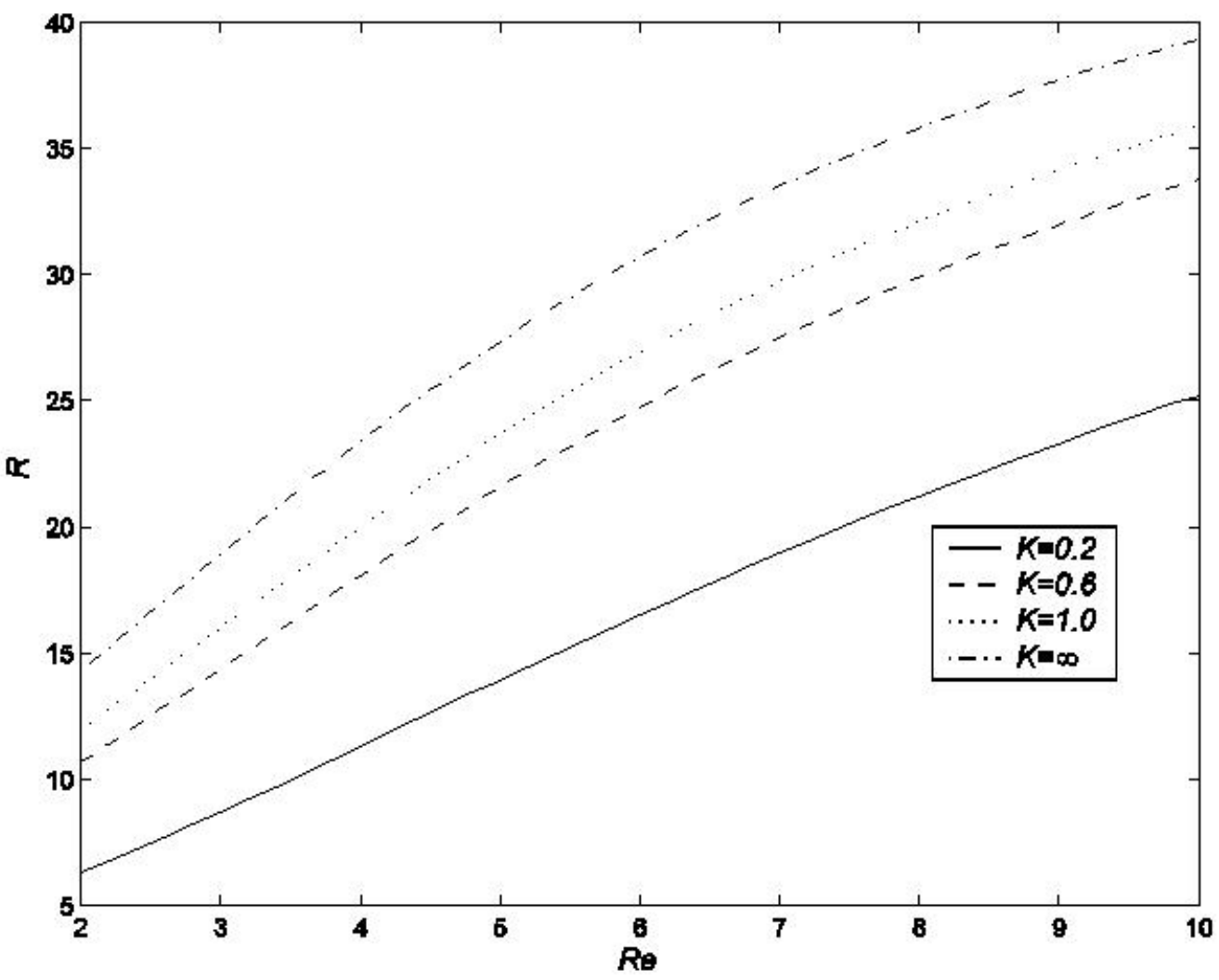

Fig.6. Amplitude of the shear stress due to primary flow for $S=1.0$.

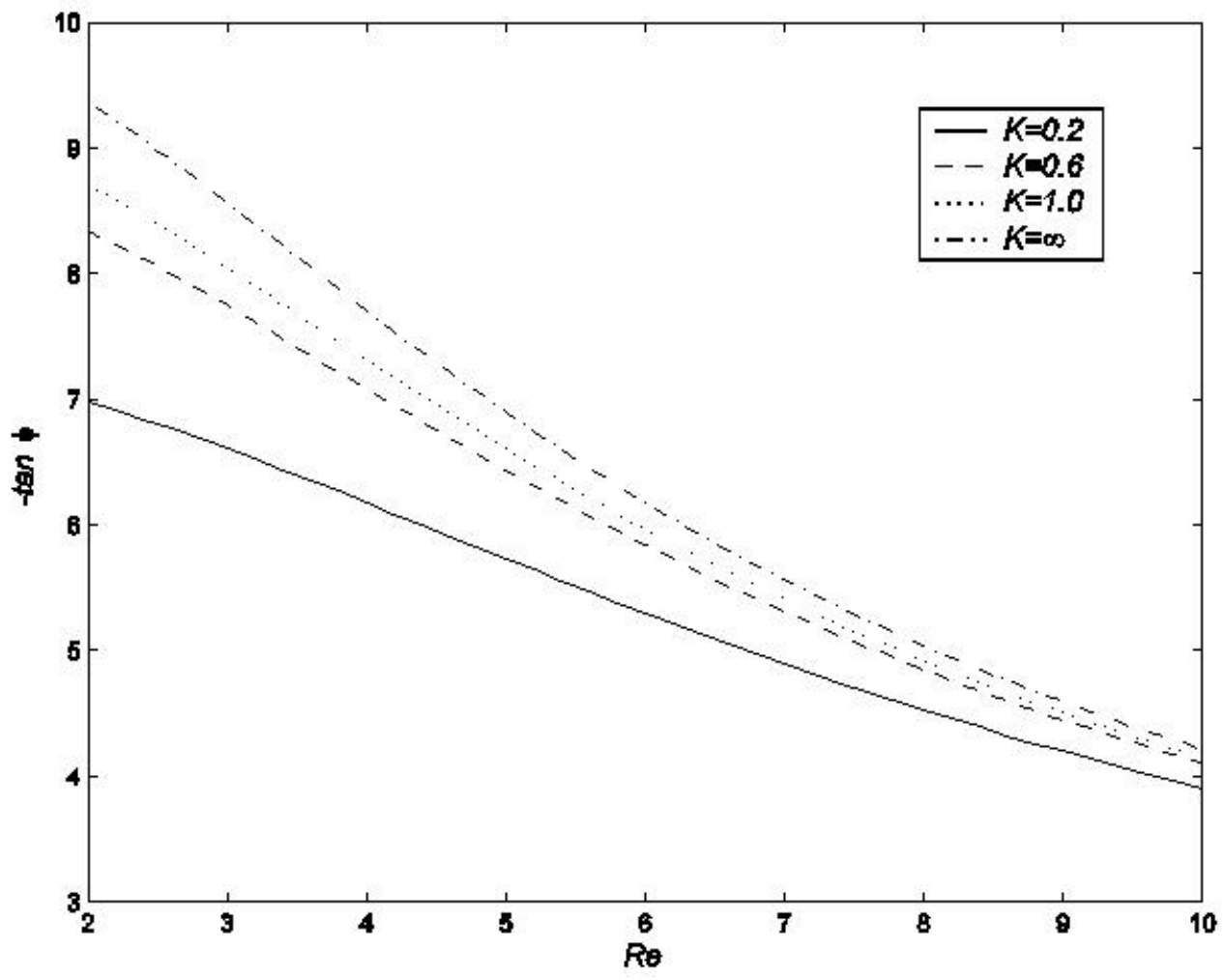

Fig.7. Tangent of phase shift of shear stress due to primary flow for $S=1.0$. 


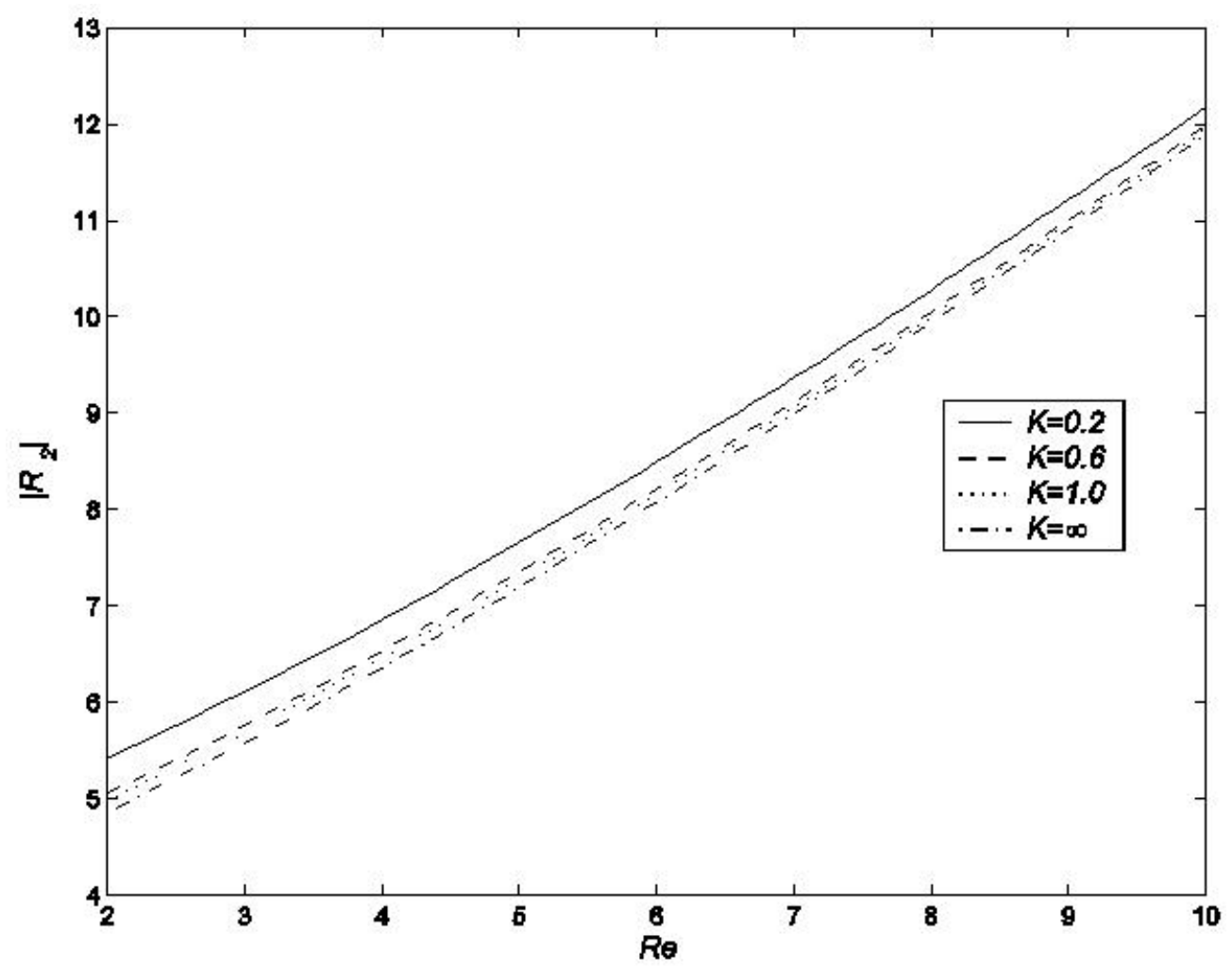

Fig.8. Shear stress due to secondary flow for $S=1.0$.

\section{Nomenclature}

$$
\begin{aligned}
C_{i}(i=1, \ldots, 10) & - \text { constants } \\
d & - \text { distance between two plates } \\
i & - \text { imaginary unity }(i=\sqrt{-1}) \\
K & - \text { non-dimensional permeability parameter } \\
K^{*} & - \text { permeability of the porous medium } \\
K_{1} & - \text { constant } \\
m_{1,2} & - \text { constants } \\
p & - \text { dimensionless pressure } \\
p & - \text { non-dimensional pressure } \\
p^{*} & - \text { pressure } \\
R & - \text { amplitude of shear stress due to primary flow } \\
R_{2} & - \text { amplitude of shear stress due to secondary flow } \\
R^{2} & - \text { Reynolds number } \\
r_{1,2} & - \text { constants } \\
S & - \text { suction parameter } \\
t & - \text { dimensionless time } \\
t^{*} & - \text { time } \\
U & - \text { free stream velocity } \\
u, v, w & - \text { non-dimensional velocity components } \\
u^{*}, v^{*}, w^{*} & - \text { velocity components } \\
&
\end{aligned}
$$




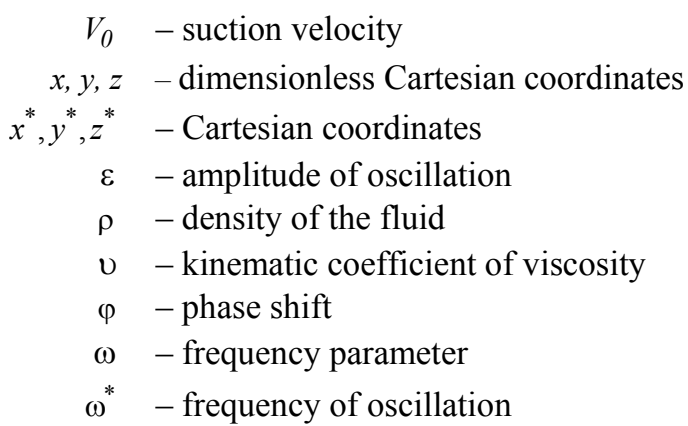

\section{References}

Guria M., Jana R.N. and Pop I. (2007): Three dimensional oscillating flow between two parallel plates with heat transfer. - International Journal of Applied Mechanics and Engineering, vol.12, No.1, pp.19-35.

Lighthill M.J. (1954): The response of laminar skin friction and heat transfer to the fluctuating in the stream velocity. Proc. Roy. Soc., vol.224A p.1-23.

Raptis A.A. (1983): Unsteady free convective flow through a porous media. - Int. J. Engng. Sci., vol.21, pp.345-348.

Raptis A.A. and Perdikis C.P. (1985): Oscillatory flow through a porous medium by the presence of free convective flow. - Int. J. Engng. Sci., vol.23, pp.51-55.

Singh K.D. and Sharma R. (2001): Three-dimensional Couette flow through a porous medium with heat transfer. Indian J. Pure Appl. Math., vol.32(12), pp.1819-1829.

Singh K.D. and Sharma R. (2002): Three-dimensional free convective flow and heat transfer through a porous medium with periodic permeability. - Indian J. Pure Appl. Math., vol.33(6), pp.941-949.

Varshney C.L. (1979): Fluctuating flow of viscous fluid through a porous medium bounded by a porous plate. - Indian J. Pure Appl. Math., vol.10 pp.1558-64.

Received: March 19, 2012

Revised: September 3, 2013 